\title{
A Method for Comprehensive Analysis of Urinary Acylglycines by Using Ultra-Performance Liquid Chromatography Quadrupole Linear Ion Trap Mass Spectrometry
}

\author{
Avalyn E. Lewis-Stanislaus and Liang Li \\ Department of Chemistry, University of Alberta, Edmonton, Alberta, Canada
}

\begin{abstract}
Acylglycines are an important class of metabolites that have been used in the diagnosis of several inborn errors of metabolism (IEM). However, current analytical methods detect only a few acylglycines. There is a need to profile these metabolites in a comprehensive manner for studying their functions and improving their diagnostic values for different IEM and potentially other diseases. We describe a sensitive method that combines the chromatographic resolving power of ultra-performance liquid chromatography (UPLC) to separate closely related metabolites including isomers with tandem mass spectrometry (MS/MS). Acylglycines were extracted from urine using an anion exchange solid-phase extraction (SPE) cartridge. After UPLC separation, the acylglycines were detected on a hybrid triple quadrupole linear ion trap mass spectrometer. A set of standards were used for the development of an optimal MS acquisition method. Several acquisition modes using information derived from collisioninduced dissociation breakdown curves were used to detect acylglycines. Using this method, 18 acylglycines were detected in the urine of healthy individuals and confirmed using standards, while 47 additional acylglycines were detected and tentatively identified, based on their retention and fragmentation pattern. Among the 65 acylglycines detected, only 18 of them have been previously reported in biofluids of healthy individuals. These results will be deposited in a public human metabolome database. This example illustrates that by developing a method tailored to the analysis of a class of metabolites sharing similar structural moieties, we can potentially identify many more new metabolites, thereby expanding the overall metabolome coverage. (J Am Soc Mass Spectrom 2010, 21, 2105-2116) (C) 2010 American Society for Mass Spectrometry
\end{abstract}

$\mathrm{M}$ etabolomics is an emerging field that is poised to play a significant role in many disciplines of biosciences. One of the current challenges in metabolomics is to profile the metabolome in a very comprehensive manner, ideally covering the entire set of metabolites present in a biological system. However, due to technical limitations, only a small portion of the metabolome is analyzed. There is a great need to expand the metabolome coverage to reveal subtle changes of the metabolome in biological studies or disease biomarker discovery. Liquid chromatography mass spectrometry (LC/MS) is a sensitive technique that can detect many metabolites in a metabolome sample [1]. However, metabolite identification from the mass spectral data alone is often difficult [2-6]. There are only a limited number of metabolite standards available for spectral comparison to identify unknowns. For example, in the Human Metabolome Database (HMDB), product ion spectra of about 900 known standards obtained by

Address reprint requests to Professor L. Li, Department of Chemistry, University of Alberta, Edmonton, Alberta T6G 2G2, Canada. E-mail: Liang.Li@ualberta.ca tandem mass spectrometry (MS/MS) are included. To create this MS/MS spectral library, we went through almost all the possible commercial sources to acquire the available metabolite standards [7]. This number is still quite small compared to the size of the human metabolome; the total number of human metabolites is unknown, but the HMDB contains over 8000 entries of endogenous human metabolites. We need to expand this spectral library to include many more metabolites found in biological sources, such as human biofluids. A comprehensive metabolite spectral library will enable many metabolomics researchers to take advantage of the database resource for identifying metabolites for biological studies or biomarker discovery.

We are currently pursuing a strategy to expand the metabolome coverage and the spectral library by systematically detecting and identifying metabolites sharing one or more similar structural moiety, such as glycine. Because some standards from this group are available, analyzing the unknown metabolites of the same group becomes more manageable. In this work, we demonstrate the development and application of this strategy to detect and identify unknown acylgly- 
cines. Acylglycines are an important class of metabolites that have been used in the diagnosis of several inborn errors of metabolism (IEM) [8].

IEMs are inherited disorders that are due to gene defects coding specific enzymes involved in the metabolism of amino acids or organic acids [8]. One of the main classes of IEMs consists of disorders of fatty acid oxidation and mitochondrial metabolism. In individuals with fatty acid oxidation disorder, a wide array of symptoms is due to the toxic fatty acid acyl-coA esters that accumulate in the mitochondria. Glycine conjugation with these acyl-coA esters has been of clinical interest due to their role in the detoxification process. Increased concentration of urinary acylglycines is generally indicative of IEMs [9-11].

The most widely used analytical methods for determining acylglycines in urine include solvent extraction, derivatization, followed by separation and detection using gas chromatography mass spectrometry (GC-MS) [12-17]. Although this established method offers many advantages, it requires specific derivatization reactions for analyzing the polar acylglycines. Also diagnoses of some IEMs can be challenging due to the relatively low sensitivity of the analytical process [18]. The application of tandem mass spectrometry (MS/MS) has been used as an alternative to GC-MS and as a high-throughput method. Analysis by fast atom bombardment (FAB) and lately electrospray ionization (ESI) have been well documented [19-22]. Although these methods can detect a larger number of disorders in a single run, they lack the ability to distinguish between isomers and detect relatively low abundance acylglycines, thus may not be able to distinguish between certain disorders [22].

We report a LC-MS method based on the use of triple quadrupole linear ion trap (QTRAP) mass spectrometer that offers higher sensitivity and more data-dependent scanning modes than a conventional triple quadrupole tandem mass spectrometer [23]. Separation is carried out by using ultra-performance liquid chromatography (UPLC), which allows for high-resolution separation of isomers and closely related acylglycines. The aim of this work is to identify as many unknown acylglycines as possible to expand the overall molecular coverage of this important class of metabolites. We show that the described method provides a sensitive analytical procedure to detect previously undetected acylglycines in the urine of healthy individuals; 65 acylglycines were found using the new method, compared to the currently known 18 compounds.

\section{Experimental}

\section{Chemicals and Reagents}

All chemicals except those noted were purchased from Sigma-Aldrich Canada (Oakville, ON, Canada). Human pooled liver microsomes and nicotinamide adenine dinucleotide phosphate (NADPH) regeneration solutions $\mathrm{A}$ and $\mathrm{B}$ were purchased from BD Gentest
(Franklin Lakes, NJ, USA). Optima grade methanol and water, Optima LCMS grade acetonitrile, and acetic anhydride and pyridine were purchased from Fisher Scientific (Ottawa, ON, Canada). HPLC-grade formic acid and ammonium hydroxide were obtained from Fluka (Milwaukee, WI, USA). The standard acylglycines used were dimethylglycine, phenylglycine, acetylglycine, propionylglycine, isobutyrylglycine, butyrylglycine, 4-hydroxyphenylacetylglycine, 2-methylbutyrylglycine, isovalerylglycine, valerylglycine, tiglyglycine, 3-methylglycine, suberylglycine, glutarylglycine, phenyacetylglycine, phenylpropionylglycine, hexanoylglycine, octanoylglycine, and hippuric acid.

\section{Samples}

Urine was collected from six healthy volunteers who were not on any special diet. An informed consent was obtained from the volunteer and ethics approval for this work was obtained from the University of Alberta in compliance with the University Health Information Act. The volunteers were all adults, ranging in age from 24 to $38 \mathrm{y}$ old. The urine samples were collected as first morning void samples for all six volunteers and urine was also collected over $12 \mathrm{~h}$ for two of those individuals. The samples were centrifuged, aliquoted, and stored at $-20{ }^{\circ} \mathrm{C}$ without any preservatives added until analysis. For long-term storage, the samples were kept at $-80^{\circ} \mathrm{C}$.

\section{Microsome Incubation}

Acylglycines standards $(50 \mu \mathrm{M})$ were individually incubated with human liver microsomes $(2 \mathrm{mg} / \mathrm{mL})$ in $100 \mathrm{mM}$ potassium phosphate, $\mathrm{pH}$ 7.4. The standards were first pre-incubated at $37^{\circ} \mathrm{C}$ for $5 \mathrm{~min}$, followed by the addition of NADPH regenerating solution, containing components $\mathrm{A}\left(26.1 \mathrm{mM} \mathrm{NADP}{ }^{+}, 66 \mathrm{mM}\right.$ glucose6-phosphate, and $66 \mathrm{mM} \mathrm{MgCl}{ }_{2}$ in $\left.\mathrm{H}_{2} \mathrm{O}\right)$ and $\mathrm{B}(40$ $\mathrm{U} / \mathrm{mL}$ glucose-6-phosphate dehydrogenase in $5 \mathrm{mM}$ sodium citrate) in $100 \mathrm{mM}$ potassium phosphate, $\mathrm{pH}$ 7.4. The solutions were incubated, with shaking, in a $37^{\circ} \mathrm{C}$ incubator for periods of $30 \mathrm{~min}, 1,3,6$, and $24 \mathrm{~h}$. Control incubations omitting either standards, NADPH regenerating solution or microsomes were also performed by substituting an equal volume of potassium phosphate buffer. The reactions were then terminated by the addition of $5 \%$ acetic acid in acetonitrile (vol/ vol). The samples were centrifuged at 14,000 $g$ for $5 \mathrm{~min}$ and the supernatants were subjected to solid-phase extraction (SPE).

\section{Solid Phase Extraction}

Oasis mixed-mode anion exchange (MAX) cartridges (Waters, Mississauga, ON, Canada) were operated at a flow rate of $1 \mathrm{~mL} / \mathrm{min}$ using a vacuum manifold (Alltech from Fisher Scientific, Ottawa, ON, Canada). 
The $30-\mathrm{mg}$ or $60-\mathrm{mg}$ cartridges were preconditioned with $1 \mathrm{~mL}$ acetonitrile followed by equilibration with 1 $\mathrm{mL}$ water. Urine $(1 \mathrm{~mL})$ was loaded onto the columns

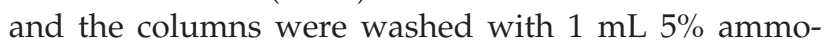
nium hydroxide solution. Acylglycines were eluted in two fractions, $1 \mathrm{~mL} 2 \%$ formic acid in $40 \%$ acetonitrile/ $60 \%$ water followed by $1 \mathrm{~mL} 2 \%$ formic acid in acetonitrile. The eluents were evaporated to dryness, using a vacuum centrifuge concentrator, and reconstituted in $100 \mu \mathrm{L} 4 \%$ acetonitrile, $0.1 \%$ formic acid in water. Samples extracted were urine, urine spiked with standards, and microsomal incubations.

\section{Esterification}

To confirm the identification of acylglycines found, methylation and acetylation were performed [20]. These reactions were optimized using standards and confirmed to be useful in assisting in compound identification. For the urine samples, after extraction of the samples by SPE, the eluents were evaporated to dryness in a vacuum centrifuge concentrator. Three hundred microliters of $3 \mathrm{M}$ methanolic $\mathrm{HCl}$ was added to the dried extract in a reaction vial and allowed to react at $65^{\circ} \mathrm{C}$ for $15 \mathrm{~min}$. The reaction mixture was then divided into two equal portions; one portion was evaporated under a stream of nitrogen and reconstituted in $100 \mu \mathrm{L}$ 10:90 acetonitrile:water ( $\mathrm{vol} / \mathrm{vol}), 0.1 \%$ formic acid. The other portion was also evaporated under a stream of nitrogen and treated with $100 \mu \mathrm{L}$ of 50:50 acetic anhydride/pyridine (vol/vol) and allowed to react at room temperature for $1 \mathrm{~h}$. The solvents were evaporated under a stream of nitrogen and the residue was reconstituted in $100 \mu \mathrm{L}$ 10:90 acetonitrile:water, $0.1 \%$ formic acid.

\section{UPLC Separation}

Chromatographic separation was done on a Waters ACQUITY UPLC system with a $1.7-\mu \mathrm{m}$ bridged ethylene hybrid $(\mathrm{BEH}), 150 \mathrm{~mm} \times 1.0 \mathrm{~mm} \mathrm{C}_{18}$ column. The column was maintained at ambient temperature. Elution was done according to the following method: $100 \%$ A for $11 \mathrm{~min}$, then a linear gradient of $0-35 \%$ B over 50 min, $35 \%-100 \%$ B over $5 \mathrm{~min}$, and held at $100 \%$ B for 9 min, where mobile phase A consisted of $4 \%$ acetonitrile, $0.1 \%$ formic acid in water, and B consisted of $0.1 \%$ formic acid acetonitrile. The flow rate of the method was $0.050 \mathrm{~mL} / \mathrm{min}$ and $5.0 \mu \mathrm{L}$ of each sample was injected onto the column.

\section{Mass Spectrometry}

Mass analysis was carried out in an ABI 4000 QTRAP mass spectrometer equipped with a TurbolonSpray source (Applied Biosystems, Foster City, CA, USA). The UPLC and mass spectrometer were both controlled by Analyst software ver.1.5 from Applied Biosystems. The mass spectrometer was operated in both positive and negative electrospray ionization (ESI) mode. General mass spectrometric conditions were: for positive mode, spray voltage, $4800 \mathrm{~V}$; temperature, $200{ }^{\circ} \mathrm{C}$; GS1, 40; GS2, 10; curtain gas, 10; CAD, high; declustering potential, 35; collision energy, $22 \mathrm{eV}$; for negative mode: spray voltage, $-3100 \mathrm{~V}$; temperature, $200^{\circ} \mathrm{C}$; GS1, 40; GS2, 10; curtain gas, 10 ; $\mathrm{CAD}$, high; declustering potential, -40 ; collision energy, $-20 \mathrm{eV}$. The acquisition method consisted of several information dependent acquisition (IDA) scan cycles including precursor ion, constant neutral loss or multiple reaction monitoring (MRM) as the survey scan and four dependent enhanced product ion (EPI) scans. IDA criteria were set to allow the four most intense peaks to trigger the EPI scans. The EPI scans were in the range of 50-500 Da, scanned at 250, 1000 , and $4000 \mathrm{Da} / \mathrm{s}$ and source parameters were the same as mentioned above. The total duty cycle was $\sim 1.2 \mathrm{~s}$. Data were collected in profile mode and analyzed using Analyst ver. 1.5 and LightSight ver. 2.0.

\section{Breakdown Graphs and Scan Modes}

To obtain optimal conditions for the detection of acylglycines, breakdown curves from collision-induced dissociation (CID) of precursor ions were constructed. Solutions of each acylglycine standard were dissolved in water at a concentration of $0.5 \mathrm{mM}$ and stored at $-20^{\circ} \mathrm{C}$. These solutions were diluted to $10 \mu \mathrm{M}$ in a mixture of $\mathrm{CH}_{3} \mathrm{CN} / \mathrm{H}_{2} \mathrm{O}$ (50:50), $0.1 \%$ formic acid and infused into the ion source with a syringe pump at a rate of $10 \mu \mathrm{L} / \mathrm{min}$. Product ion spectra were obtained from the precursor ion in the positive mode by averaging 30 cycles. Scans were performed at different collision energies ranging from 5 to $40 \mathrm{eV}$, with a step size of $5 \mathrm{eV}$.

Based on the information obtained from the breakdown curves, appropriate neutral loss and precursor ion scans were performed as survey scans in IDA experiments, which triggered four dependent EPI scans. MRM transitions were set up based on masses of acylglycines that were detected in the neutral loss and precursor ion scans and those acylglycines likely to be found in urine but whose signals were too low to be detected by the constant neutral loss and precursor ion scans. IDA experiments were created with these MRM transitions as a survey scan, in an experiment similar to the precursor and neutral loss methods.

\section{Results and Discussion}

\section{Sample Handling Issues}

The main purpose of this work was to develop a means of detecting as many acylglycines as possible. We thus optimized each step of the analysis procedure to maximize the performance of the technique. For example, current methods of extracting acylglycines from urine include liquid-liquid extraction followed by derivatization $[12,13]$. However, human urine is a complex 
mixture of salts, hydrophilic and hydrophobic compounds, peptides and proteins. In this work, a more selective sample extraction method was developed. To optimize the solid-phase extraction procedure, two cartridges, hydrophilic-lipophilic balanced (HLB) and mixed-mode anion-exchange (MAX) cartridges, were tested. Factors to optimize the conditions include composition, volumes and $\mathrm{pH}$ of the wash and eluting solutions (data not shown). MAX cartridge was chosen based on its high selectivity in retaining the acylglycines. The optimal conditions are listed in the experimental section. This method was found to be effective in isolating acylglycines and organic acids from human urine; for acylglycine standards, recoveries of greater than $88 \%$ were obtained. The extraction method was also reproducible. As an example, four urine samples were extracted using four different SPE cartridges and were injected into the UPLC to compare the performance of the different MAX cartridges. The results shown in Supplemental Figure S1, which can be found in the electronic version of this article, indicate excellent cartridge-to-cartridge reproducibility, signifying the robustness of the extraction procedure.

Another improvement is in the area of LC separation. Instead of HPLC, UPLC was used in this work. UPLC capitalizes on the use of sub-2- $\mu$ m particles to offer superior efficiency and resolution compared to HPLC [24]. The higher efficiency translates into better detection sensitivity with narrower peaks which increases the detectability of acylglycines present in low concentrations in urine. The higher resolution is signif- icant in separating isomeric and isobaric species. It was found that the UPLC method used was efficient in separating the isomers of acylglycines, which is very important in aiding the assignment of potential structures to unknown acylglycines. An example of the high-resolution separation is shown in Supplemental Figure S2. Baseline separation can be observed for all isomers of C5 and C5:1. In contrast, HPLC could not resolve these peaks well and some of the low intensity peaks were not observed (data not shown).

\section{MS Fragmentation Patterns of Standards}

Analyzing the MS fragmentation patterns of available standards can facilitate the development and optimization of a MS/MS method for sensitive and selective detection of a group of similar compounds including unknowns. In this work, seventeen acylglycines were used to generate the MS/MS fragmentation information. The fragmentation pathways of acylglycines are shown in Scheme 1 and the pathways of the glycineconjugated dicarboxylic acids (DCs) are shown in Supplemental Scheme S1. As these schemes show, common neutral losses of masses $18\left(\mathrm{H}_{2} \mathrm{O}\right), 46\left(\mathrm{H}_{2} \mathrm{O}+\mathrm{CO}\right), 75$ $\left(\mathrm{NH}_{2} \mathrm{CH}_{2} \mathrm{COOH}\right)$, and $103\left(\mathrm{NH}_{2} \mathrm{CH}_{2} \mathrm{COOH}+\mathrm{CO}\right)$ are observed for most of the acylglycines. Less common fragments observed are the neutral losses of $60\left(\mathrm{CH}_{2}=\right.$ $\left.\mathrm{C}(\mathrm{OH})_{2}\right), 93\left(\mathrm{NH}_{2} \mathrm{CH}_{2} \mathrm{COOH}+\mathrm{H}_{2} \mathrm{O}\right)$, observed in straight chain acylglycines longer than six carbons and $117\left(\mathrm{CH}_{2}=\mathrm{C}(\mathrm{OH}) \mathrm{NHCH}_{2} \mathrm{COOH}\right)$, which is observed as a major fragment in phenypropionylglycine, result-<smiles></smiles>
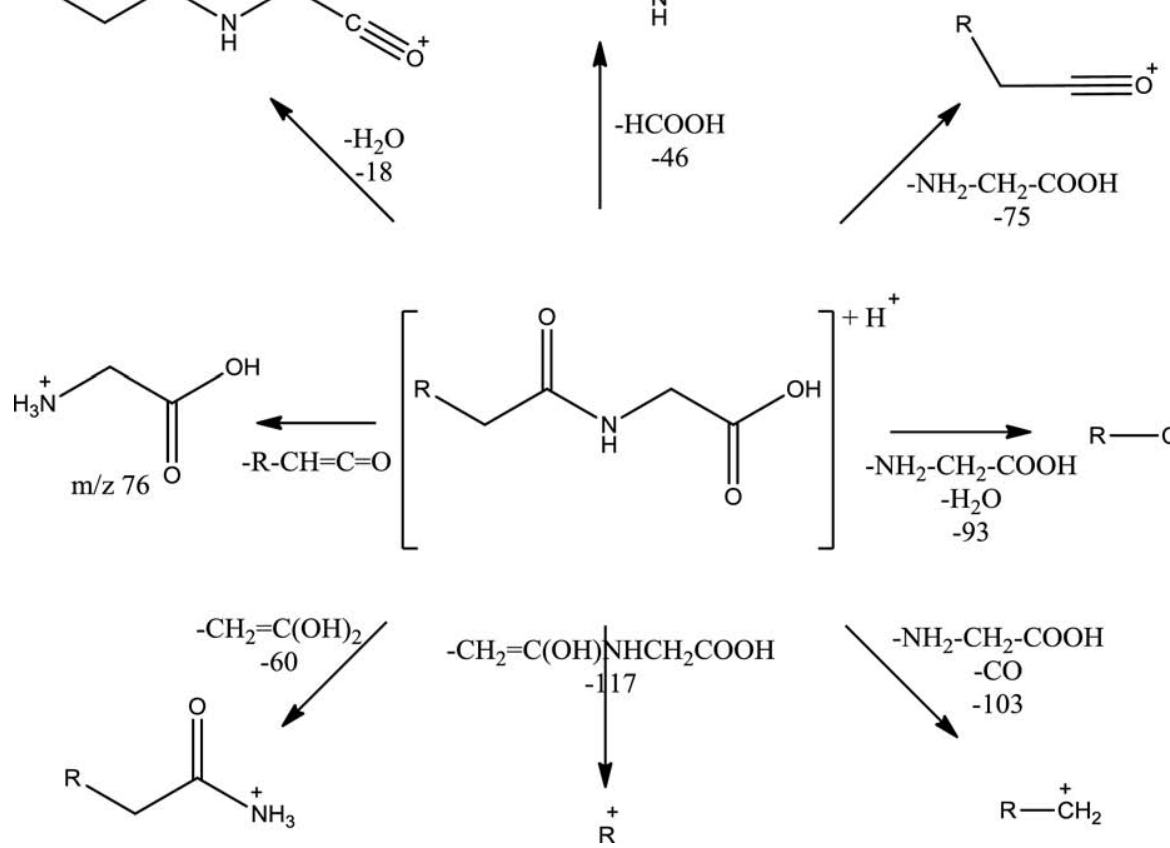

Scheme 1. Proposed fragmentation pathways of acylglycines. 
ing in the stable tropyllium ion. Many acylglycines also show a major fragment ion at $\mathrm{m} / \mathrm{z} 76$, which corresponds to the protonated glycine $\left(\mathrm{H}_{3}{ }^{+} \mathrm{NCH}_{2} \mathrm{COOH}\right)$. This fragmentation pathway occurs because aliphatic acylglycines lose the acyl moiety as a ketene. There must be an available proton on carbon 2 of the acyl group in order for this fragmentation to occur [19].

Fragmentation in QTRAP can be induced either inside the linear ion trap or in the collision cell in the triple quadrupole mode. The instrument is capable of all the scans common to the triple quadrupole instrument, such as precursor scanning (PC), constant neutral loss scanning (NL) and multiple-reaction monitoring (MRM), as well as trap scan modes enhanced product ion (EPI), enhanced mass (EMS), and enhanced resolution (ER) scans. The instrument can also perform both quadrupole and trap scans in a single run and switching from one to the other only takes a few ms. This can be done in an information dependent data acquisition (IDA), which combines two or more scan modes in a single LC-MS/MS run. The mass spectrometer switches from the first scan or a survey scan usually done as an MS scan to a second data dependent MS/MS scan when an eluting peak rises above a predetermined threshold level. In this work, MRM, constant neutral loss scanning and precursor scanning were used as survey scans with the enhanced product ion scans as the dependent MS/MS scans.

Currently, all acylglycines detected by direct infusion ESI-MS/MS use a precursor of $m / z 76$ method or if methylation is done, a precursor of $\mathrm{m} / \mathrm{z} 90$ method [20]. In our work, the optimal energetic conditions for the detection of acylglycines were obtained from the breakdown curves. The best choice for collision energy and MRM transitions can be readily observed from these curves. A typical breakdown curve in this work is shown in Figure 1a for valeryglycine. Most of the typical losses (as shown in Scheme 1) are observed. At low to medium collision energy $(15<\mathrm{CE}<30)$, the most abundant fragment ion is $\mathrm{m} / \mathrm{z} 76$ and at high collision energy ( $\mathrm{CE}>30)$, the fragment ion corresponding to the loss of 103 is the most abundant.

Although most acylglycines yield a fragment at $\mathrm{m} / \mathrm{z}$ 76, not all of them do. The breakdown curve of tiglycine, shown in Figure 1b, demonstrates this. The major loss observed is the loss of $75 \mathrm{Da}$, the neutral glycine moiety. No fragment ion at $m / z 76$ is generated because there is no available proton on carbon 2 of the acyl group [19, 22]. An IDA method, which uses a survey scan of neutral loss of 75, should be used to detect acylgycines, including tiglyglycine, 3-methycrotonylglycine, hippuric acid, and hydroxyhippuric acid isomers, among others. These acylglycines would not be detected using a precursor of $\mathrm{m} / \mathrm{z} 76$ method. Therefore the optimal transition for MRM is precursor ion fragmentation corresponding to the loss of $75 \mathrm{Da}$.

It is important to note that the fragment ion at $m / z 76$ may not be the most intense fragment in the product ion spectra and if this transition is used in detection, sensitivity is lost. Examples of this are shown in Figure 1c and d. Breakdown curves of aromatic acylglycines reveal that the neutral loss of 103 is the most abundant fragment and not the fragment at $\mathrm{m} / \mathrm{z} 76$. The optimal conditions for the detection of aromatic acylglycines at
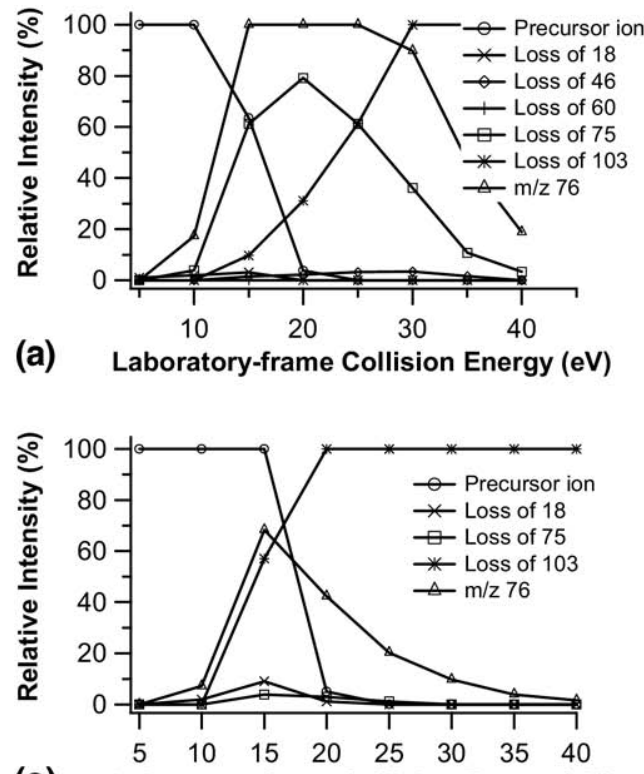

(c) Laboratory-frame Collision Energy (eV)

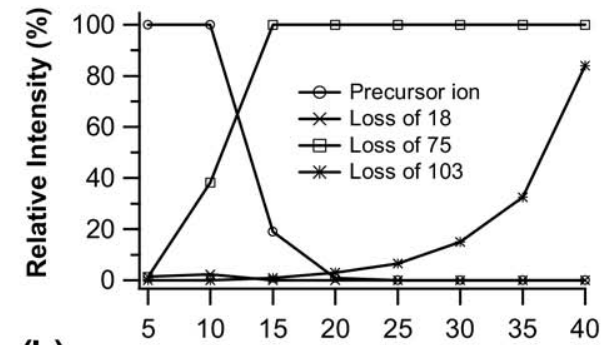

(b) Laboratory-frame Collision Energy (eV)

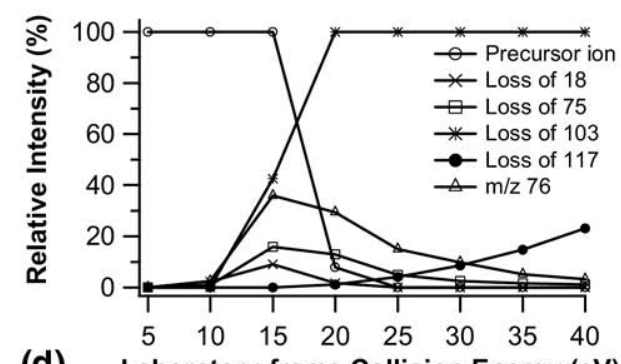

(d)

Laboratory-frame Collision Energy $(\mathrm{eV})$

Figure 1. Breakdown graphs of (a) valerylglycine, (b) tiglyglycine, (c) phenylacetylglycine, and (d) phenylpropionylglycine. Fragmentation studies were performed using a 4000 QTRAP instrument. Low-energy CID was performed with nitrogen as the collision gas. The source parameters were optimized and operating conditions were as follows: CAD gas $=$ nitrogen; spray voltage $=4800 \mathrm{~V}$; temperature $=200{ }^{\circ} \mathrm{C} ; \mathrm{GS} 1=40 ; \mathrm{GS} 2=10 ;$ curtain gas $=10 ; \mathrm{CAD}=$ high; $\mathrm{DP}=35$. 
a CE of 20 is an MRM transition from the precursor ion to the fragment ion produced with the loss of 103 (e.g., for phenylacetylglycine, $m / z 194 \rightarrow 91$ ).

Other trends observed in the fragmentation of acylglycines are the following: losses of 18 and 46 are usually low in intensity and sometimes not observed, especially in aromatic glycines and glycines conjugated to unsaturated fatty acids. Fragment ion $m / z 76$ is the major fragment for straight and branched chain acylglycines. Loss of 103 is more intense in branched chains than in straight chains, especially when it is branched at the 2-position of the fatty acid chain. For the glycine conjugates of dicarboxylic acid, the fragment ion at $\mathrm{m} / \mathrm{z}$ 76 is not the major product ion. In this case, loss of 64 fragment ion is the most abundant fragment ion at a CE of $20 \mathrm{eV}$. Losses of 18 and 46 are higher in intensities than in the acylglycines.

\section{MS Scan Modes for Sample Analysis}

Because most acylglycines yield an intense fragment ion at $m / z 76$ in the positive mode, an IDA experiment was performed using precursor scanning as a survey scan followed by enhanced product ion scan as the data dependent MS/MS scan. Acylglycines also possess a carboxylic acid group, so they can be analyzed in the negative ionization mode. In the negative ion mode they lose the deprotonated glycine moiety $\left(\mathrm{H}_{2} \mathrm{NCH}_{2} \mathrm{COO}^{-}, \mathrm{m} / z\right.$ 74). A similar IDA experiment was performed with a precursor of $m / z 74$ scan as the survey scan. A comparison of the positive and negative precursor scans is shown in Figure 2a,b. Negative ion scan did not significantly improve the selectivity and sensitivity in comparison to the positive mode. Most acylglycines were detected in both modes and the overall intensities were quite similar. Some acylglycines that do not generate a fragment ion at $m / z 76$ will still generate the fragment ion at $\mathrm{m} / \mathrm{z} 74$, such as tiglyglycine and 3-methycrotonylglycine. Several earlier eluting peaks like acetylglycine, isobutyrylglycine, butyrylglycine, and hydroxyphenylacetylglycine were detected at considerably higher intensities using the positive ionization mode. Due to this and the fact that positive MS/MS scans give much more structural information than those acquired in negative mode, all subsequent scans were done in the positive ion mode.

As mentioned earlier, some acylglycines do not fragment to yield a product ion at $m / z$ 76. However, all acylglycines commonly lose the neutral glycine fragment (a neutral loss of $75 \mathrm{Da}$ ) and the remaining acyl fragment retains the positive charge. An IDA experiment in the positive ionization mode, similar to that described above, was performed, except the survey scan used was a neutral loss of $75 \mathrm{Da}$ scan. Figure 2c shows a total ion chromatogram of an IDA experiment using constant neutral loss of $75 \mathrm{Da}$ as the survey scan. Acylglycines such as hippuric acid, 3-methylcrotonylglycine, tiglyglycine, phenylglycine, hydroxyhippuric acid, and methylhippuric acid were not detected in the
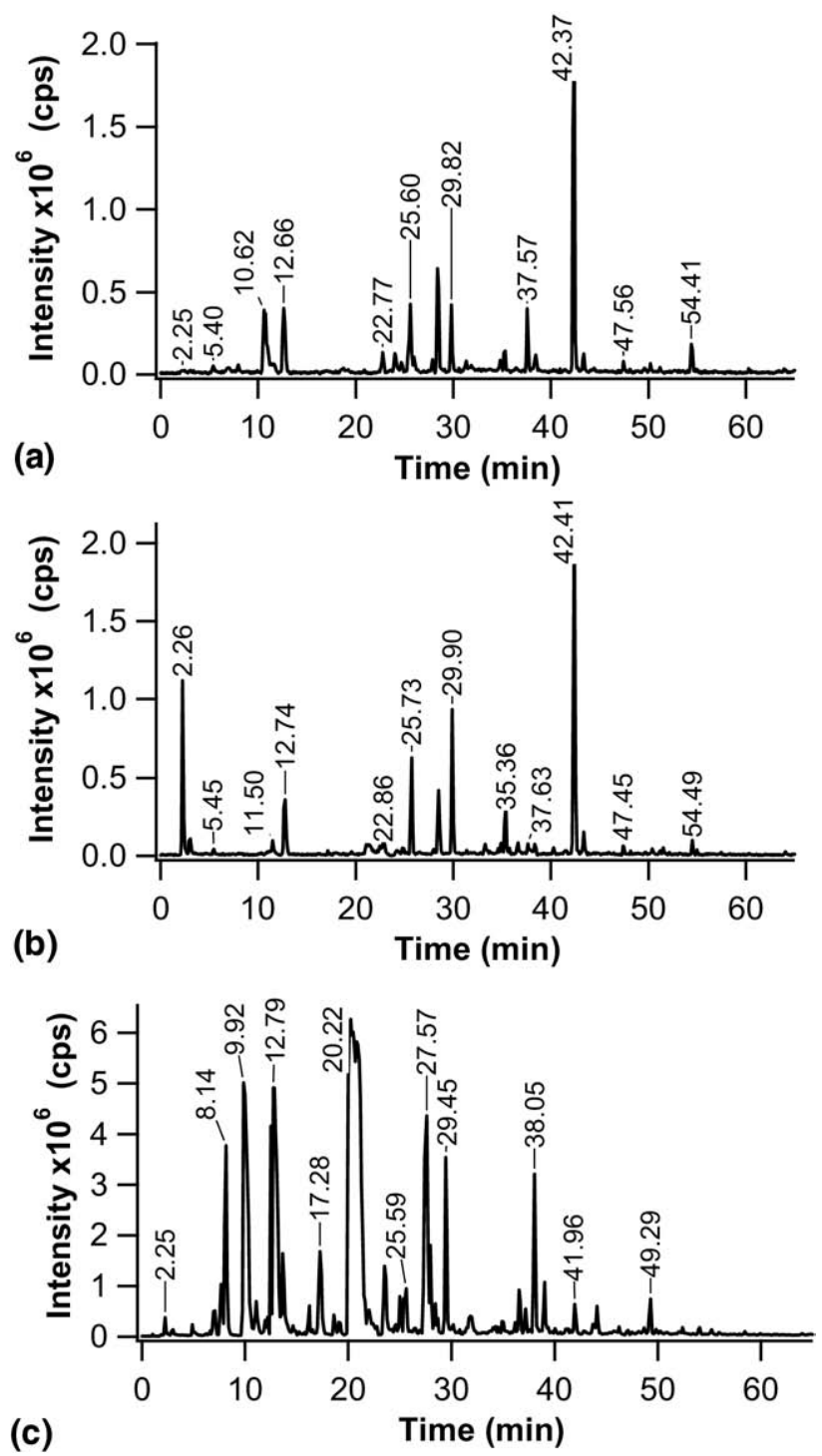

Figure 2. Ion chromatograms from UPLC MS/MS analysis of healthy human urine: (a) TIC of precursor ion scanning of $\mathrm{m} / \mathrm{z} 74$ (negative mode), (b) TIC of precursor ion scanning of $\mathrm{m} / \mathrm{z} 76$ (positive mode), and (c) TIC of neutral loss scanning of 75 Da. Urine sample from a healthy volunteer was analyzed using three information dependent acquisition (IDA) methods of precursor ion scans in the positive and negative modes and neutral loss scan in the positive mode.

precursor method and exhibited major fragment ions at $\mathrm{m} / \mathrm{z} 105,83,83,77,121$ and 119 , respectively, corresponding to the neutral loss of $75 \mathrm{Da}$ (see the Supplemental Data for all the MS/MS spectra of acylglycines). Comparison of the TICs shown in Figure $2 b$ and $c$ shows that most of the acylglycines observed in the precursor method were also found using the neutral loss method. However, the neutral loss method was not as selective because other classes of compounds that gave the neutral loss of $75 \mathrm{Da}$ (the identities of these compounds were unknown) were also detected and many acylglycines were detected at lower intensities.

When targeting certain compound families in biological matrices, high selectivity and sensitivity become 
very essential. Precursor and constant neutral loss scans are highly selective, but are only moderately sensitive. A much better alternative is to use MRM as the survey scan to trigger the enhanced product ion data collection. MRM is much more selective and sensitive than either precursor scanning or constant neutral loss scanning. Due to the differences in the fragmentation of certain acylglycines, specific MRM transitions were chosen for each acylglycine using fragmentation information gleaned from the precursor scanning and constant neutral loss scanning methods and the breakdown curves. The precursor scanning and constant neutral loss scanning survey scans provided information about the masses of all possible acylglycines that can be observed and the breakdown curves were used to provide the best possible transitions. These optimized MRM transitions as well as dwell times and collision energy are shown in Table 1. The survey scan contained 62 MRM transitions and each transition was performed with a dwell time of $15 \mathrm{~ms}$ and a CE of $20 \mathrm{eV}$. The scan time was $1.2 \mathrm{~s}$ for all transitions. The IDA intensity threshold was set to 100 counts per second (cps) and the dependent scans were performed in enhanced product ion mode with a CE of $20 \mathrm{eV}$. Four enhanced product ion scans were performed on the four most intense peaks before switching back to MRM scan mode.

\section{Human Urine Analysis}

After the method was developed, we applied it to analyze human urine samples using the 62 MRM transitions to detect acylglycines. Figure 3 shows the ion chromatograms obtained from UPLC MS/MS of urine samples of six healthy volunteers. Relative differences in intensities of acylglycines can be observed. Most of acylglycines are common to all six individuals with the exception of dimethylglycine and 4-hydroxyphenylacetylglycine, which are only detected in four and five individuals, respectively. Acylglycine identification was confirmed using standards and tentative identification of other acylglycines was done using retention time and class-specific fragmentation patterns. To identify an unknown acylglycine, at least four of seven neutral losses must be observed. Water loss is very common and not considered to be structurally valuable.

Due to the lack of standards, positive identification of many unknown acylglycines cannot be made. We have attempted to use the methods of human liver microsome (HLM) metabolite production and chemical derivatization to provide tentative identification for some of the unknowns. In the HLM method, individual acylglycine standards were incubated with human liver microsomes to generate the metabolites of acylglycines

Table 1. MRM scanning conditions used in the analysis of acylglycines in the positive ion mode

\begin{tabular}{|c|c|c|c|c|c|c|c|}
\hline $\mathrm{Q} 1(\mathrm{~m} / \mathrm{z})$ & Q3 $(\mathrm{m} / \mathrm{z})$ & Dwell time (ms) & $\mathrm{CE}(\mathrm{eV})$ & Q1 $(m / z)$ & O3 $(\mathrm{m} / \mathrm{z})$ & Dwell time (ms) & $\mathrm{CE}(\mathrm{eV})$ \\
\hline 104 & 58 & 15 & 20 & 204 & 76 & 15 & 20 \\
\hline 118 & 76 & 15 & 20 & 204 & 140 & 15 & 20 \\
\hline 146 & 76 & 15 & 20 & 206 & 76 & 15 & 20 \\
\hline 152 & 77 & 15 & 20 & 206 & 103 & 15 & 20 \\
\hline 158 & 83 & 15 & 20 & 208 & 105 & 15 & 20 \\
\hline 160 & 76 & 15 & 20 & 210 & 107 & 15 & 20 \\
\hline 162 & 76 & 15 & 20 & 212 & 137 & 15 & 20 \\
\hline 170 & 95 & 15 & 20 & 214 & 76 & 15 & 20 \\
\hline 172 & 75 & 15 & 20 & 214 & 111 & 15 & 20 \\
\hline 172 & 69 & 15 & 20 & 216 & 76 & 15 & 20 \\
\hline 174 & 76 & 15 & 20 & 216 & 123 & 15 & 20 \\
\hline 176 & 76 & 15 & 20 & 218 & 76 & 15 & 20 \\
\hline 176 & 64 & 15 & 20 & 218 & 125 & 15 & 20 \\
\hline 180 & 105 & 15 & 20 & 218 & 154 & 15 & 20 \\
\hline 181 & 106 & 15 & 20 & 222 & 76 & 15 & 20 \\
\hline 184 & 109 & 15 & 20 & 222 & 119 & 15 & 20 \\
\hline 186 & 76 & 15 & 20 & 224 & 76 & 15 & 20 \\
\hline 186 & 83 & 15 & 20 & 224 & 121 & 15 & 20 \\
\hline 188 & 76 & 15 & 20 & 226 & 76 & 15 & 20 \\
\hline 188 & 95 & 15 & 20 & 232 & 168 & 15 & 20 \\
\hline 190 & 76 & 15 & 20 & 242 & 149 & 15 & 20 \\
\hline 190 & 126 & 15 & 20 & 242 & 167 & 15 & 20 \\
\hline 194 & 91 & 15 & 20 & 244 & 169 & 15 & 20 \\
\hline 194 & 119 & 15 & 20 & 246 & 76 & 15 & 20 \\
\hline 196 & 121 & 15 & 20 & 246 & 182 & 15 & 20 \\
\hline 198 & 76 & 15 & 20 & 250 & 147 & 15 & 20 \\
\hline 198 & 95 & 15 & 20 & 256 & 76 & 15 & 20 \\
\hline 200 & 76 & 15 & 20 & 256 & 163 & 15 & 20 \\
\hline 200 & 97 & 15 & 20 & 258 & 194 & 15 & 20 \\
\hline 202 & 76 & 15 & 20 & 260 & 196 & 15 & 20 \\
\hline 202 & 109 & 15 & 20 & 276 & 201 & 15 & 20 \\
\hline
\end{tabular}



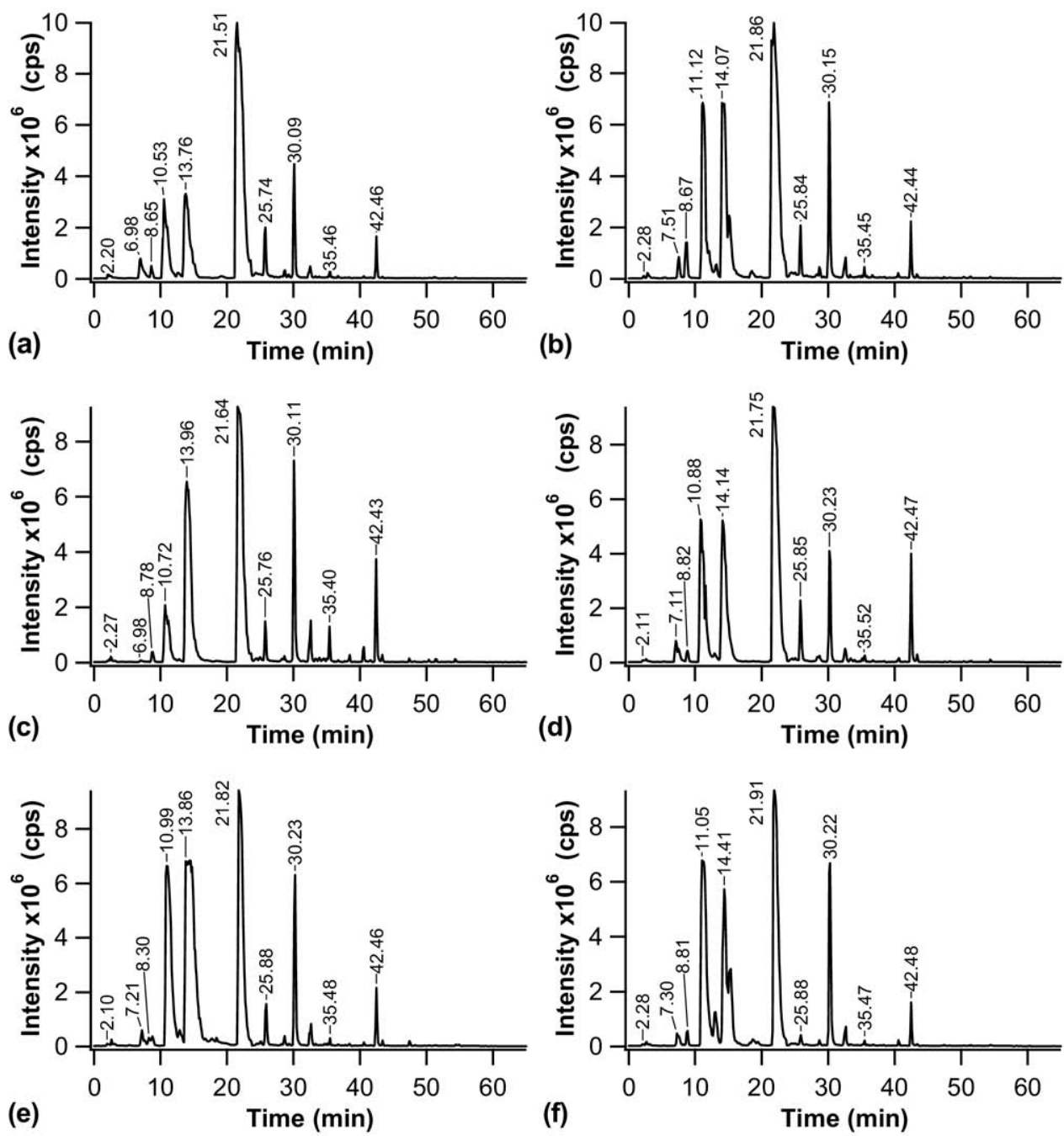

Figure 3. Ion chromatograms from UPLC MS/MS analysis of six healthy human urine samples obtained by using multiple reaction monitoring scans with 62 diagnostic transitions. Urine samples were collected as first morning void from six volunteers eating normal diets.

potentially found in human urine. The hydroxylated and carbonyl-substituted metabolites were the most common metabolites observed in the microsome incubations. A comparison of the retention times and fragmentation patterns of the metabolites was used to aid in the identification of some of the unknown acylglycines. An example of such a comparison is shown in Figure 4. The ion chromatograms from microsome incubation and urine are shown in Figure 4a and c, respectively. Figure $4 \mathrm{~b}$ shows the MS/MS spectrum from the chromatographic peak labeled with a diamond at the retention time of $21.11 \mathrm{~min}$ (Figure $4 \mathrm{a}$ ). Figure $4 \mathrm{~d}$ shows the MS/MS spectrum from the peak with a diamond at the retention time of $21.22 \mathrm{~min}$ (Figure 4c). The inset in Figure $4 \mathrm{~d}$ shows the fragmentation pattern of hydroxyphenylpropionylglycine. Comparison of the MS/MS spectra shows losses of $18,46,75,103$ and 117 in the spectrum of the urine sample. Losses of 18 and 46 (not diagnostic) and the precursor ion are not observed in the MS/MS spectrum of the microsome extract (possibly too high $\mathrm{CE}$ ), but losses of 75, 103, and 117 are common to both, with the major fragment ion being the loss of 117. Even though not all the fragment ions are observed, the diagnostic losses are common. Comparison of retention time and MS/MS spectra allows for the identification of hydroxyphenylpropionylglycine. It is important to note that the isomers generated by the microsomes are not necessarily the same isomers excreted in urine but the isomeric information is nonetheless valuable.

Chemical derivatization is also useful for acylglycine identification. We note that compounds other than acylglycines can also produce a fragment ion of $\mathrm{m} / \mathrm{z} 76$ in the product ion scan. However, comparison between underivatized urine and derivatized urine can assist in identifying acylglycines and distinguish them from other compounds. A shift of $14 \mathrm{Da}$ in the precursor mass (28 Da in the case of DCs), an appropriate shift in 

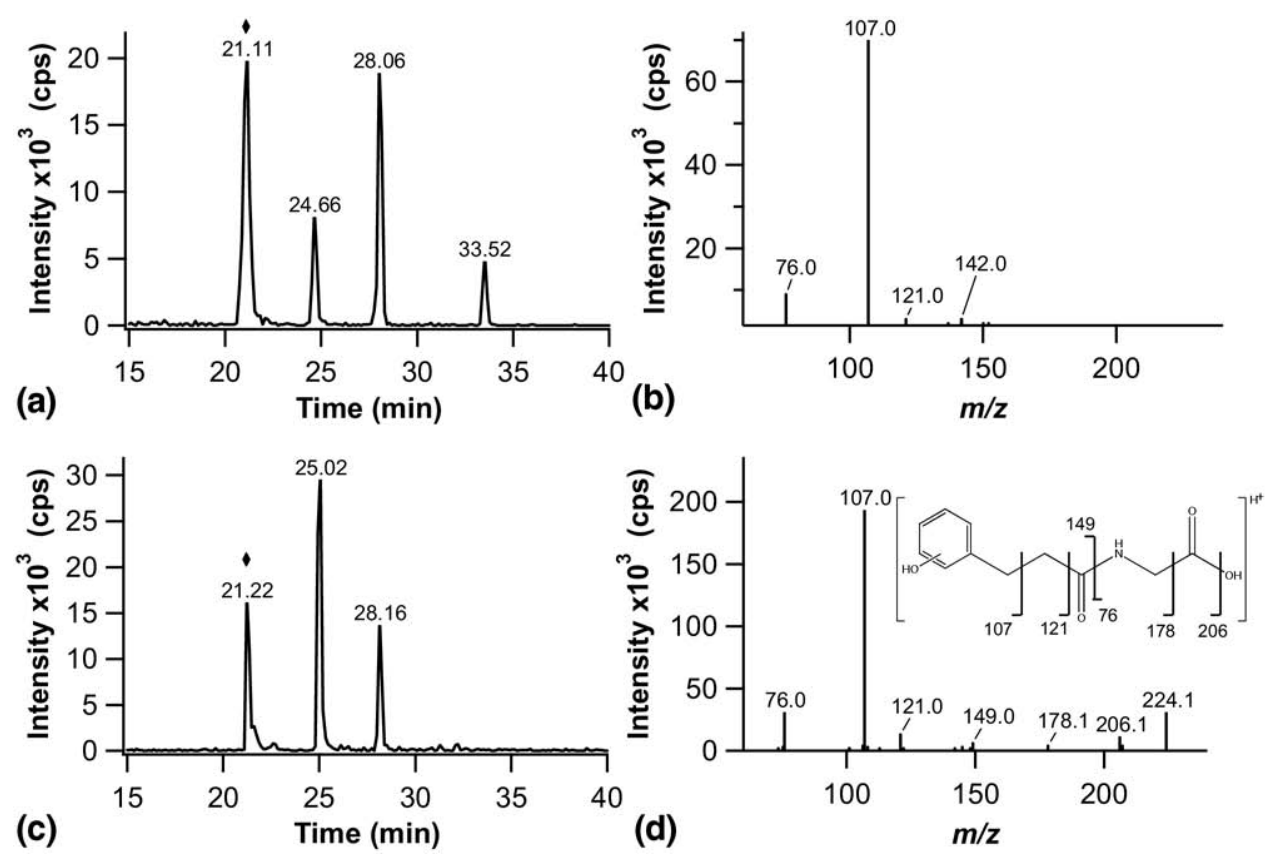

Figure 4. Comparison of extracted ion chromatograms of $\mathrm{m} / \mathrm{z} 224$ (hydroxyphenylpropionylglycine) extracted from microsomes (a) and urine (c), and corresponding MS/MS spectra, (b) and (d). The inset in (d) shows the fragmentation pattern of hydroxyphenylpropionylglycine.

retention time and corresponding losses in the product ion scans should be observed for acylglycines. For example, C6:1 acylglycine, previously unreported in the urine of healthy individuals, was confirmed as a acylglycine by methylation and the results are shown in Supplemental Figure S3.

We have detected a total of 65 acylglycines and they are listed in Table 2 in three groups (positively identified, tentatively identified, and unknown structures). Only 18 of them have been previously reported. All of the acylglycines used in screening for fatty acid disorders were detected with the exception of propionylglycine. Propionylglycine was not observed in any of the individuals but was detected in low levels in a spiked urine sample (the concentration of the spiked propionylglycine was $10 \mu \mathrm{M}$ ); this compound appears to be present in very low concentrations in urine of healthy individuals.

The standard deviations of the chromatographic retention times of acylglycines detected from different individuals were calculated and also presented in Table 2. This information is useful for other users who use similar separation conditions for analyzing these metabolites. The MS/MS spectra of these compounds are provided as Supplemental Material and will be deposited to a free, public-accessible database (www.hmdb. ca) [7]. This database has over 8000 entries of mainly endogenous human metabolites, including MS/MS spectra of about 900 metabolites.

While Figure 3 illustrates that many acylglycines can be detected from urine samples of different individuals, a similar number of acylglycines can also be detected from urine samples of an individual collected at differ- ent time points. This is illustrated in a preliminary work where we examined the effect of the time of sampling on the acylglycine profile of urine. For urine sample collection, the most common type of collection is the first morning voiding. Often a sample is collected during fasting because analytes of interest may be excreted in higher concentrations. However, other conditions that occur in fasting can lead to an abnormal excretion of other metabolites. In this study, urine was collected from a healthy individual at the first morning voiding, for five consecutive days (to assess the effect of diet), at $4 \mathrm{~h}$ after a heavy meal and also was collected over $12 \mathrm{~h}$. Figure 5 shows the ion chromatograms of urine collected at the first morning voiding from an individual from five consecutive days. An overlay of total ion chromatograms of multiple reaction monitoring scans using 62 diagnostic transitions is shown. The ion chromatograms of other urine samples collected are shown in Supplemental Figure S4. Results of this comparison study revealed very little differences in the acylglycine profile. Relative intensities may differ between time collections but the same acylglycines were detected in all the time collections.

The above examples demonstrate that a large number of acylglycines can be consistently detected from urine samples collected from different individuals or from the same individual at different times. Thus, the developed method should be suitable for profiling many acylglycines for potential discovery of new biomarkers for IEMs and other diseases by using a large pool of samples with clinical information on the individuals. Work in this direction is planned. 
Table 2. A list of confirmed and tentatively identified acylglycines present in urine of six healthy volunteers $(n=6)^{*}$

\begin{tabular}{|c|c|c|c|}
\hline Confirmed acylglycines $(n=6)$ & $m / z$ & $\mathrm{RT}(\min )$ & References \\
\hline \multicolumn{4}{|l|}{ Acylglycine } \\
\hline Dimethylglycineł & 104 & $2.12 \pm 0.0$ & {$[25,26]$} \\
\hline Phenylglycine & 152 & $2.26 \pm 0.06$ & \\
\hline Acetylglycine & 118 & $2.37 \pm 0.06$ & {$[19,20]$} \\
\hline Isobutyrylglycine & 146 & $5.44 \pm 0.13$ & {$[13,27]$} \\
\hline 4-Hydroxyphenylacetylglycinet & 210 & $10.22 \pm 0.18$ & \\
\hline 2-Methylbutyrylglycine & 160 & $11.12 \pm 0.12$ & {$[13,28,29]$} \\
\hline Tiglyglycine & 158 & $11.89 \pm 0.19$ & {$[14,30-32]$} \\
\hline Isovalerylglycine & 160 & $12.86 \pm 0.16$ & {$[13,14,17,33,34]$} \\
\hline 3-Methylcrotonylglycine & 158 & $12.97 \pm 0.19$ & {$[14,30,35]$} \\
\hline Valerylglycine & 160 & $16.38 \pm 0.18$ & \\
\hline Hippuric acid & 180 & $22.21 \pm 0.04$ & {$[15,30,36-38]$} \\
\hline Suberylglycine & 232 & $25.43 \pm 0.06$ & {$[13,17]$} \\
\hline Phenylacetylglycine (PAG) & 194 & $25.88 \pm 0.06$ & {$[20]$} \\
\hline Hexanoylglycine & 174 & $30.07 \pm 0.04$ & {$[13,14,17,19]$} \\
\hline Phenylpropionylglycine (PPG) & 208 & $33.88 \pm 0.04$ & [13] \\
\hline Octanoylglycine & 202 & $51.16 \pm 0.03$ & \\
\hline \multicolumn{4}{|l|}{ Tentatively identified acylglycines } \\
\hline Malonylglycine/hydroxybutyrylglycine (or isomers)† & 162 & $2.59 \pm 0.05$ & \\
\hline Succinylglycine/hydroxyvalerylglycine (or isomers) & 176 & $4.42 \pm 0.14$ & \\
\hline Dihydroxyhippuric acid (hydroxyls positions unknown) & 212 & $5.27 \pm 0.06$ & \\
\hline Dihydroxyhippuric acid (hydroxyls positions unknown) & 212 & $6.98 \pm 0.22$ & \\
\hline 2-Furoylglycine & 170 & $7.20 \pm 0.19$ & {$[30,38,39]$} \\
\hline Dihydroxyhippuric acid (hydroxyls positions unknown) & 212 & $8.24 \pm 0.27$ & \\
\hline Hydroxyhippuric acid (hydroxyl position unknown) & 196 & $8.77 \pm 0.06$ & {$[21,30,4043]$} \\
\hline Dihydroxyhippuric acid (hydroxyls positions unknown) & 212 & $10.85 \pm 0.23$ & \\
\hline Hydroxyhippuric acid (hydroxyl position unknown) & 196 & $10.91 \pm 0.26$ & {$[21,30,3240]$} \\
\hline Hydroxyhexanoylglycine (hydroxyl position unknown) & 190 & $11.61 \pm 0.16$ & \\
\hline 2-Pentenoylglycine & 158 & $13.85 \pm 0.17$ & \\
\hline Hydroxyhippuric acid (hydroxyl position unknown) & 196 & $14.19 \pm 0.27$ & {$[30,40]$} \\
\hline Nicotinuric acid & 181 & $15.03 \pm 0.21$ & {$[41,42]$} \\
\hline Hydroxyphenylacetylglycinet (hydroxyl position unknown) & 210 & $15.74 \pm 0.26$ & \\
\hline Hydroxyphenylpropionylglycine (hydroxyl position unknown) & 224 & $21.37 \pm 0.16$ & \\
\hline Hydroxyphenylpropionylglycine (hydroxyl position unknown) & 224 & $22.68 \pm 0.09$ & \\
\hline Hexenoylglycine (double bond position unknown) & 172 & $23.06 \pm 0.07$ & \\
\hline Hexenoylglycine (double bond position unknown) & 172 & $24.53 \pm 0.05$ & \\
\hline Hydroxyphenylpropionylglycine (hydroxyl position unknown) & 224 & $28.16 \pm 0.04$ & \\
\hline Methylvalerylglycine (methyl position unknown) & 174 & $28.65 \pm 0.03$ & \\
\hline Heptenoylglycine (double bond position unknown) & 186 & $28.71 \pm 0.03$ & \\
\hline Hydroxyoctenoylglycine (hydroxyl position unknown) & 216 & $28.93 \pm 0.03$ & \\
\hline Methylhippuric acid (methyl position unknown) & 194 & $32.58 \pm 0.05$ & {$[30,43,44]$} \\
\hline Hydroxyoctanoylglycine (hydroxyl position unknown) & 218 & $33.31 \pm 0.04$ & \\
\hline Octadienoylglycine (double bonds positions unknown) & 198 & $35.07 \pm 0.03$ & \\
\hline Hydroxyoctanoylglycine (hydroxyl position unknown) & 218 & $35.46 \pm 0.03$ & \\
\hline Octadienoylglycine (double bonds positions unknown) & 198 & $36.75 \pm 0.03$ & \\
\hline Sebacylglycine & 260 & $38.12 \pm 0.06$ & \\
\hline Methylhexanoylglycine (methyl position unknown) & 188 & $38.47 \pm 0.02$ & \\
\hline Heptanoylglycine & 188 & $41.07 \pm 0.04$ & \\
\hline Octadienoylglycine (double bonds positions unknown) & 198 & $41.40 \pm 0.01$ & \\
\hline Phenylbutyrylglycine & 222 & $41.61 \pm 0.05$ & \\
\hline Cis-3,4-Methylene-heptanoylglycine & 200 & $42.46 \pm 0.02$ & \\
\hline Octenoylglycine (double bond position unknown) & 200 & $43.38 \pm 0.02$ & \\
\hline Nonenoylglycine (double bond position unknown) & 214 & $47.44 \pm 0.01$ & \\
\hline Nonanoylglycine & 216 & $54.95 \pm 0.02$ & \\
\hline \multicolumn{4}{|l|}{ Unknown acylglycines } \\
\hline Unknown \#1 & 210 & $30.41 \pm 0.03$ & \\
\hline Unknown \#2 & 242 & $32.90 \pm 0.03$ & \\
\hline Unknown \#3 & 188 & $35.22 \pm 0.03$ & \\
\hline Unknown \#4‡ & 258 & $35.29 \pm 0.06$ & \\
\hline Unknown \#5 & 200 & $35.45 \pm 0.02$ & \\
\hline Unknown \#6 & 244 & $36.28 \pm 0.03$ & \\
\hline Unknown \#7 & 256 & $38.06 \pm 0.03$ & \\
\hline Unknown \#8 & 242 & $40.27 \pm 0.04$ & \\
\hline Unknown \#9 & 222 & $50.27 \pm 0.04$ & \\
\hline
\end{tabular}


Table 2. Continued

\begin{tabular}{lll}
\hline Confirmed acylglycines $(n=6)$ & $m / z$ & RT (min) \\
\hline \hline Unknown \#10 & 226 & $52.60 \pm 0.03$ \\
Unknown \#11 & 224 & $54.39 \pm 0.03$ \\
Unknown \#12 & 250 & $56.84 \pm 0.03$ \\
Unknown \#13 & 256 & $66.54 \pm 0.02$ \\
\hline
\end{tabular}

* Compounds marked with ${ }^{\dagger}$ are only found in five individuals and those marked with ${ }^{\ddagger}$ are only found in four individuals. References quoted are for acylglycines detected in healthy individuals by other methods. The MS/MS spectra of all the listed compounds can be found in the supplemental data.

\section{Conclusions}

We have developed a method for the comprehensive analysis of acylglycines in the urine of healthy subjects. Putative identification of acylglycines was achieved using retention time information and characteristic fragmentation patterns. Derivatization or esterification was not necessary to enhance detection, when using selective extraction methods like SPE and sensitive detection instruments like the hybrid QTRAP mass spectrometer. Greater selectivity and sensitivity were achieved using MRM scans and simultaneous product ion scans provide structural elucidation. The use of optimal acquisition modes is very important. Constant neutral loss and precursor methods are valuable for detection of wide range of acylglycines and for the discovery of expected as well as unexpected, or previously undetected, acylglycines. Breakdown curves provided optimal transitions and conditions, as well as important trends to note for detection of classes of acylglycines. With this strategy, detection of expected acylglycines was enhanced and novel acylglycines were discovered.

A total of 65 acylglycines were detected and of those only 15 are used for diagnosis of metabolic diseases. Additional studies need to be done using clinical samples of subjects with known IEMs or other diseases to determine the effectiveness of the method in the diagnoses of these disorders. The dataset generated in this study will be significant in future research to further

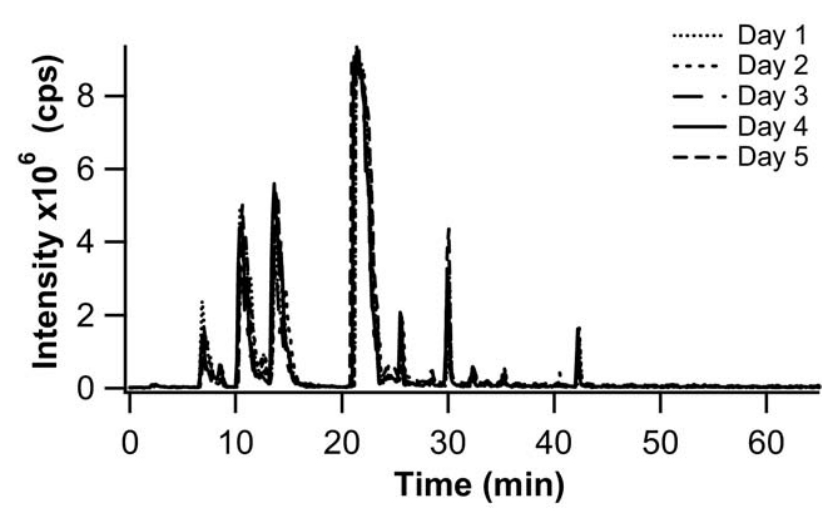

Figure 5. Ion chromatograms from UPLC MS/MS analysis of urine collected as first morning void from a healthy individual for $5 \mathrm{~d}$. An overlay of total ion chromatograms of multiple reaction monitoring scans using 62 diagnostic transitions is shown. understand, discover and improve the diagnosis of new inborn errors of metabolism and other metabolic diseases related to the excretion of acylglycines.

\section{Acknowledgments}

The authors acknowledge funding for this work by Genome Canada through Genome Alberta's Human Metabolomics project and the Canada Research Chairs program.

\section{Appendix A Supplementary Material}

Supplementary material associated with this article may be found in the online version at doi:10.1016/ j.jasms.2010.09.004.

\section{References}

1. Scalbert, A.; Brennan, L.; Fiehn, O.; Hankemeier, T.; Kristal, B. S.; van Ommen, B.; Pujos-Guillot, E.; Verheij, E.; Wishart, D.; Wopereis, S. Mass-Spectrometry-Based Metabolomics: Limitations and Recommendations for Future Progress with Particular Focus on Nutrition Research. Metabolomics 2009, 5, 435-458.

2. Bowen, B. P.; Northen, T. R. Dealing with the Unknown: Metabolomics and Metabolite Atlases. J. Am. Soc. Mass Spectrom. 2010, 21, 1471-1476.

3. Bullinger, D.; Fux, R.; Nicholson, G.; Plontke, S.; Belka, C.; Laufer, S.; Gleiter, C. H.; Kammerer, B. Identification of Urinary Modified Nucleosides and Ribosylated Metabolites in Humans Via Combined ESI-FTICR MS and ESI-IT MS Analysis. J. Am. Soc. Mass Spectrom. 2008, 19, 1500-1513.

4. Zhou, M.; McDonald, J. F.; Fernández, F. M. Optimization of a Direct Analysis in Real Time/Time-of-Flight Mass Spectrometry Method for Rapid Serum Metabolomic Fingerprinting. J. Am. Soc. Mass Spectrom. 2010, 21, 68-75.

5. Lin, L.-C.; Wu, H.-Y.; Tseng, V. S.-M.; Chen, L.-C.; Chang, Y.-C.; Liao, P.-C. A Statistical Procedure to Selectively Detect Metabolite Signals in LC-MS Data Based on Using Variable Isotope Ratios. J. Am. Soc. Mass Spectrom. 2010, 21, 232-241.

6. Want, E. J.; Wilson, I. D.; Gika, H.; Theodoridis, G.; Plumb, R. S.; Shockcor, J.; Holmes, E.; Nicholson, J. K. Global Metabolic. Profiling Procedures for Urine Using UPLC-MS. Nat. Protoc. 2010, 5, 1005-1018.

7. Wishart, D. S.; Knox, C.; Guo, A. C.; Eisner, R.; Young, N.; Gautam, B.; Hau, D. D.; Psychogios, N.; Dong, E.; Bouatra, S.; Mandal, R.; Sinelnikov, I.; Xia, J. G.; Jia, L.; Cruz, J. A.; Lim, E.; Sobsey, C. A.; Shrivastava, S.; Huang, P.; Liu, P.; Fang, L.; Peng, J.; Fradette, R.; Cheng, D.; Tzur, D.; Clements, M.; Lewis, A.; De Souza, A.; Zuniga, A.; Dawe, M.; Xiong, Y. P.; Clive, D.; Greiner, R.; Nazyrova, A.; Shaykhutdinov, R.; Li, L.; Vogel, H. J.; Forsythe, I. Hmdb: A Knowledgebase for the Human Metabolome. Nucleic Acids Res. 2009, 37, D603-D610.

8. Sahai, I.; Marsden, D. Newborn Screening. Crit. Rev. Clin. Lab. Sci. 2009, $46,55-82$.

9. Sim, K. G.; Hammond, J.; Wilcken, B. Strategies for the Diagnosis of Mitochondrial Fatty Acid $\beta$-Oxidation Disorders. Clin. Chim. Acta 2002, 323, 37-58

10. Hale, D. E.; Bennett, M. J. Fatty-Acid Oxidation. Disorders-a New Class of Metabolic Diseases. J. Pediat. 1992, 121, 1-11.

11. Pasquali, M.; Monsen, G.; Richardson, L.; Alston, M.; Longo, N. Biochemical Findings in Common Inborn Errors of Metabolism. Am. J. Med. Genet. Part C-Semin. Med. Genet. 2006, 142C, 64-76.

12. Kouremenos, K. A.; Pitt, J.; Marriott, P. J. Metabolic Profiling of Infant Urine Using Comprehensive Two-Dimensional Gas Chromatography: 
Application to the Diagnosis of Organic Acidurias and Biomarker Discovery. J. Chromatog. A 2010, 1217, 104-111.

13. Costa, C. G.; Guerand, W. S.; Struys, E. A.; Holwerda, U.; ten Brink, H. J.; de Almeida, I. T.; Duran, M.; Jakobs, C. Quantitative Analysis of Urinary Acylglycines for the Diagnosis of $\beta$-Oxidation Defects Using GC-NCI-MS. J. Pharmaceut. Biomed. Anal. 2000, 21, 1215-1224.

14. Hagen, T.; Korson, M. S.; Sakamoto, M.; Evans, J. E. A GC/MS/MSs Screening Method for Multiple Organic Acidemias from Urine Specimens. Clin. Chim. Acta 1999, 283, 77-88.

15. Suh, J. W.; Lee, S. H.; Chung, B. C. GC-MS Determination of Organic Acids with Solvent Extraction after Cation-Exchange Chromatography. Clin. Chem. 1997, 43, 2256-2261.

16. Carter, S. M. B.; Midgley, J. M.; Watson, D. G.; Logan, R. W. Measurement of Urinary Medium Chain Acyl Glycines by Gas Chromatography Negative-Ion Chemical Ionization Mass Spectrometry. J. Pharmaceut. Biomed. Anal. 1991, 9, 969-975.

17. Kimura, M.; Yamaguchi, S. Screening for Fatty Acid $\beta$-Oxidation Disorders-Acylglycine Analysis by Electron Impact Ionization Gas Chromatography-Mass Spectrometry. J. Chromatogr. B 1999, 731, 105110 .

18. Downing, M.; Allen, J. C.; Bonham, J. R.; Edwards, R. G.; Manning, N. J.; Olpin, S. E.; Pollitt, R. J. Problems in the Detection of Fatty Acid Oxidation Defects: Experience of a Quality Assurance Program for Qualitative Urinary Organic Acid Analysis. J. Inherited Metab. Dis. 1999, 22, 289-292.

19. Millington, D. S.; Kodo, N.; Terada, N.; Roe, D.; Chace, D. H. The Analysis of Diagnostic Markers of Genetic Disorders in Human Blood and Urine Using Tandem Mass Spectrometry with Liquid Secondary Ion Mass Spectrometry. Int. J. Mass Spectrom. 1991, 111, 211-228.

20. Bonafe, L.; Troxler, H.; Kuster, T.; Heizmann, C. W.; Chamoles, N. A.; Burlina, A. B.; Blau, N. Evaluation of Urinary Acylglycines by Electrospray Tandem Mass Spectrometry in Mitochondrial Energy Metabolism Defects and Organic Acidurias. Mol. Genet. Metab. 2000, 69, 302-311.

21. Shigematsu, Y.; Hata, I.; Tanaka, Y. Stable-Isotope Dilution. Measurement of Isovalerylglycine by Tandem Mass Spectrometry in Newborn Screening for Isovaleric Acidemia. Clin. Chim. Acta 2007, 386, 82-86.

22. Rashed, M. S. Clinical Applications of Tandem Mass Spectrometry: Ten Years of Diagnosis and Screening for Inherited Metabolic Diseases. J. Chromatogr. B Anal. Technol. Biomed. Life Sci. 2001, 758, 27-48.

23. Hopfgartner, G.; Varesio, E.; Tschappat, V.; Grivet, C.; Bourgogne, E.; Leuthold, L. A. Triple Quadrupole Linear Ion Trap Mass Spectrometer for the Analysis of Small Molecules and Macromolecules. J. Mass Spectrom. 2004, 39, 845-855.

24. Guillarme, D.; Schappler, J.; Rudaz, S.; Veuthey, J. L. Coupling UltraHigh-Pressure Liquid Chromatography with Mass Spectrometry. TracTrends Anal. Chem. 2010, 29, 15-27.

25. Laryea, M. D.; Steinhagen, F.; Pawliczek, S.; Wendel, U. Simple Method for the Routine Determination of Betaine and N,N-Dimethylglycine in Blood and Urine. Clin. Chem. 1998, 44, 1937-1941.

26. Moolenaar, S. H.; Poggi-Bach, J.; Engelke, U. F. H.; Corstiaensen, J. M. B.; Heerschap, A.; de Jong, J. G. N.; Binzak, B. A.; Vockley, J.; Wevers, R. A. Defect in Dimethylglycine Dehydrogenase, a New Inborn Error of Metabolism: NMR Spectroscopy Study. Clin. Chem. 1999, 45, $459-464$.

27. Sass, J. O.; Sander, S.; Zschocke, J. Isobutyryl-CoA Dehydrogenase. Deficiency: Isobutyrylglycinuria and Acad8 Gene Mutations in Two Infants. J. Inherit. Metab. Dis. 2004, 27, 741-745.

28. Gibson, K. M.; Burlingame, T. G.; Hogema, B.; Jakobs, C.; Schutgens, R. B. H.; Millington, D.; Roe, C. R.; Roe, D. S.; Sweetman, L.; Steiner, R. D.; Linck, L.; Pohowalla, P.; Sacks, M.; Kiss, D.; Rinaldo, P.; Vockley, J. 2-Methylbutyryl-Coenzyme a Dehydrogenase Deficiency: A New Inborn Error of L-Isoleucine Metabolism. Pediat. Res. 2000, 47, 830-833.

29. Tein, I.; Haslam, R. H. A.; Rhead, W. J.; Bennett, M. J.; Becker, L. E.; Vockley, J. Short-Chain Acyl-CoA Dehydrogenase Deficiency-a Cause of Ophthalmoplegia and Multicore Myopathy. Neurology 1999, 52, 366-372.
30. Liebich, H. M.; Forst, C. Basic Profiles of Organic-Acids in Urine. J. Chromatog. Biomed. Appl. 1990, 525, 1-14.

31. Bennett, M. J.; Powell, S.; Swartling, D. J.; Gibson, K. M. Tiglylglycine Excreted in Urine in Disorders of Isoleucine Metabolism and the Respiratory-Chain Measured by Stable-Isotope Dilution GC-MS. Clin. Chem. 1994, 40, 1879-1883.

32. Garcia-Villoria, J.; Navarro-Sastre, A.; Fons, C.; Perez-Cerda, C.; Baldellou, A.; Fuentes-Castello, M. A.; Gonzalez, I.; Hernandez-Gonzalez, A.; Fernandez, C.; Campistol, J.; Delpiccolo, C.; Cortes, N.; Messeguer, A.; Briones, P.; Ribes, A. Study of Patients and Carriers with 2-Methyl-3Hydroxybutyryl-CoA Dehydrogenase (MHBD) Deficiency: Difficulties in the Diagnosis. Clin. Biochem. 2009, 42, 27-33.

33. Rinaldo, P.; Welch, R. D.; Previs, S. F.; Schmidtsommerfeld, E.; Gargus, J. J.; Oshea, J. J.; Zinn, A. B. Ethylmalonic Adipic Aciduria-Effects of Oral Medium-Chain Triglycerides, Carnitine, and Glycine on Urinary Excretion of Organic Acids, Acylcarnitine, and Acylglycines. Pediat. Res. 1991, 30, 216-221.

34. Fries, M. H.; Rinaldo, P.; SchmidtSommerfeld, E.; Jurecki, E.; Packman, S. Isovaleric Acidemia: Response to a Leucine Load after Three Weeks of Supplementation with Glycine, L-Carnitine, and Combined GlycineCarnitine Therapy. J. Pediat. 1996, 129, 449-452.

35. Eminoglu, F. T.; Ozcelik, A. A.; Okur, I.; Tumer, L.; Biberoglu, G.; Demir, E.; Hasanoglu, A.; Baumgartner, M. R. 3-Methylcrotonyl-CoA Carboxylase Deficiency: Phenotypic Variability in a Family. J. Child Neurol. 2009, 24, 478-481.

36. Roowi, S.; Stalmach, A.; Mullen, W.; Lean, M. E. J.; Edwards, C. A.; Crozier, A. Green Tea Flavan-3-Ols: Colonic Degradation and Urinary Excretion of Catabolites by Humans. J. Agr. Food Chem. 2010, 58, 1296-1304.

37. Ahmadi, F.; Asgharloo, H.; Sadeghi, S.; Gharehbagh-Aghababa, V.; Adibi, H. Post-Derivatization Procedure for Determination of Hippuric Acid after Extraction by an Automated Micro Solid Phase Extraction System and Monitoring by Gas Chromatography. J. Chromatogr. B Anal. Technol. Biomed. Life Sci. 2009, 877, 2945-2951.

38. Liebich, H. M.; Gesele, E.; Woll, J.; Urinary Organic. Acid Screening by Solid-Phase Microextraction of the Methyl Esters. J. Chromatogr. B 1998, 713, 427-432.

39. Pettersen J. E.; Jellum, E. Identification and Metabolic Origin of 2Furoylglycine and 2,5-Furandicarboxylic Acid in Human Urine. Clin. Chim. Acta 1972, 41, 199

40. Chen, Y. F.; Sullards, M. C.; Hoang, T. T.; May, S. W.; Orlando, T. M. Analysis of Organoselenium and Organic Acid Metabolites by Laser Desorption Single Photon Ionization Mass Spectrometry. Anal. Chem. 2006, 78, 8386-8394

41. Li, A. C.; Chen, Y. L.; Junga, H.; Shou, W. Z.; Jiang, X.; Naidong, W. Separation of Nicotinic Acid and Six Metabolites within 60 Seconds Using High-Flow Gradient Chromatography on Silica Column with Tandem Mass Spectrometric Detection. Chromatographia 2003, 58, 723 731.

42. Stratford, M. R. L.; Dennis, M. F. High-Performance LiquidChromatographic Determination of Nicotinamide and Its Metabolites in Human and Murine Plasma and Urine. J. Chromatogr. Biomed. Appl. 1992, 582, 145-151.

43. Pacenti, M.; Dugheri, S.; Villanelli, F.; Bartolucci, G.; Calamai, L.; Boccalon, P.; Arcangeli, G.; Vecchione, F.; Alessi, P.; Kikic, I.; Cupelli, V. Determination of Organic Acids in Urine by Solid-Phase Microextraction and Gas Chromatography-Ion Trap Tandem Mass Spectrometry Previous with 'in Sample' Derivatization with Trimethyloxonium Tetrafluoroborate. Biomed. Chromatogr. 2008, 22, 1155-1163.

44. Ohashi, Y.; Mamiya, T.; Mitani, K.; Wang, B. L.; Takigawa, T.; Kira, S.; Kataoka, H. Simultaneous Determination of Urinary Hippuric Acid, O-, M-, and P-Methylhippuric Acids, Mandelic Acid, and Phenylglyoxylic Acid for Biomonitoring of Volatile Organic Compounds by Gas Chromatography-Mass Spectrometry. Anal. Chim. Acta 2006, 566, 167-171. 